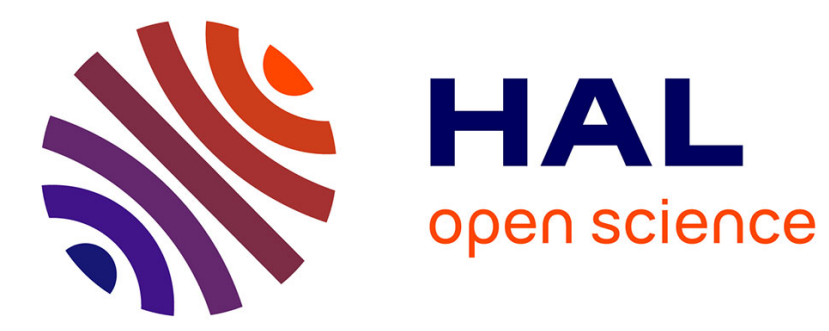

\title{
Improve the Accessibility of Tactile Charts
}

Christin Engel, Gerhard Weber

\section{To cite this version:}

Christin Engel, Gerhard Weber. Improve the Accessibility of Tactile Charts. 16th IFIP Conference on Human-Computer Interaction (INTERACT), Sep 2017, Bombay, India. pp.187-195, 10.1007/9783-319-67744-6_12 . hal-01676157

\section{HAL Id: hal-01676157 \\ https://hal.inria.fr/hal-01676157}

Submitted on 5 Jan 2018

HAL is a multi-disciplinary open access archive for the deposit and dissemination of scientific research documents, whether they are published or not. The documents may come from teaching and research institutions in France or abroad, or from public or private research centers.
L'archive ouverte pluridisciplinaire HAL, est destinée au dépôt et à la diffusion de documents scientifiques de niveau recherche, publiés ou non, émanant des établissements d'enseignement et de recherche français ou étrangers, des laboratoires publics ou privés. 


\title{
Improve the Accessibility of Tactile Charts
}

\author{
Christin Engel and Gerhard Weber \\ TU Dresden, Faculty of Computer Science, Chair of Human-Computer Interaction, \\ 01062 Dresden \\ \{christin.engel, gerhard.weber\}@tu-dresden.de
}

\begin{abstract}
Blind and visually impaired people are able to access visual charts by the mean of tactile representations. However, their production is time-consuming and requires know-how and skills in tactile chart design. Our main goal is to support blind, visually impaired as well as sighted authors by automating the creation process of tactile charts. We follow an user-centered design approach. Therefore, we analyzed both transcribers and users of tactile charts by conducting a survey on blind, visually impaired and sighted authors. On that basis, we identified steps and challenges of the production process, how users interact with tactile charts, design guidelines, as well as user preferences and tasks. As a result, we summarize requirements for an application that automates the creation of tactile charts.
\end{abstract}

Keywords: tactile charts; audio-tactile charts; tactile graphics; accessibility; effective design; blind users; survey; user requirements

\section{Introduction}

Information graphics, visual charts in particular, were developed for the visual sense and make use of domain-specific skills. Due to the preattentive perception, the user gets a quick overview of the data, detects patterns and outliers quickly [10]. There are several approaches to enable blind and visually impaired people access information graphics. Verbal descriptions are often used to get non-visual access to visual charts $[4,2,11]$. The development of those descriptions takes a high effort and, furthermore, it is time-consuming to read them out. They only cover the description of visual and structural properties of a chart such as labels, axis scale or number of bars. Some approaches focus on textual descriptions which include high-level content of the data visualization, too, in order to provide an idea of chart's intention such as Demir et al. [5] and Moraes et al. [12]. Tactile charts may be more effectively for analyzing data than verbal descriptions or data tables [15].

Tactile charts consist of raised elements, for instance lines, areas and symbols which can be perceived by the sense of touch. Colors are replaced by a variety of different textures and line styles to distinguish chart elements. Several guidelines relate to the design of tactile graphics, in particular [6,3], because visual and tactile charts differ in their design [8]. The development of tactile charts is very 
time-consuming and requires knowledge about tactile chart design [14]. Some approaches are intended to facilitate the creation of tactile charts for different use cases. Jayant et al. [9] developed the Tactile Graphics Assistant (TGA) that support direct translation of a visual into a tactile chart with image processing methods. Such kind of tools require a well-designed visual chart that follows general design conventions. However, a direct translation may not be appropriate for an effective tactile chart design [7,13]. Initial prototypes, for example the tactile chart generation tool developed by Goncu et al. [7] or the tool by Araki et al. [1] show the need for automating the creation of tactile charts. Nevertheless, the majority of existing tools only support a few chart types. The resulting charts developed by Watanabe et al. [15] are inflexible in their design because the application just supports customizing labels and raw data input. Current approaches do not support rich design such as Goncu et al.'s [7] tool that offers just one layout for bar charts and one for pie charts. The design of tactile charts heavily depends on specific use cases and user preferences.

Basically, an initial survey on the production of tactile graphics in Germany delivers indications about current practice and user experiences in exploring and creating tactile graphics [14]. However, this survey focuses on tactile graphics in general. Needs for tactile charts may differ. As a consequence we have instigated our own survey. We present the results as well as requirements for a software that automates the creation of accessible charts.

\section{Survey on Tactile Charts}

Our survey addresses blind, visually impaired and sighted authors as well as users of tactile charts. The survey was distributed by the means of more than 100 e-mails to media centers, schools, research institutes and online libraries. In addition, an invitation to the survey was published by online newsletters of three associations for blind and visually impaired people ${ }^{1}$.

The survey consists of three parts: The first part comprises 6 to 16 questions (depends on given answers) concerning experiences in chart production. In the second part, participants were asked 11 questions about their experiences in exploring and current practice in designing tactile charts. Each part starts with a filter question. The last part includes demographic data such as age, professionals, visual abilities or Braille reading skills.

\subsection{Participants}

In total, 71 users of tactile charts ( 34 blind, 7 visually impaired, 30 sighted) with an average age of 43 years completed our survey. All blind (except three who gave no answer) and none of the sighted participants have Braille skills. The majority of the participants (in total 60) use tactile charts in the context of their

\footnotetext{
${ }^{1}$ http://www.bbsb.org

http://www.isar-projekt.de

http://www.bfs-nrw-ev.de
} 
profession. They are from more than 40 institutions for instance schools (48\%), universities $(17 \%)$ or media center (14\%). Eight participants work or study in university. Figure 1 summarizes the work description of the participants. Most of them are teachers (20 participants). Independently from profession the majority of creators have also experiences in exploring tactile charts (44 out of 53).

The next sections present the results of our survey. Section 2.2 focuses on the actual chart production process, whereas Section 2.3 summarizes user requirements and preferences.

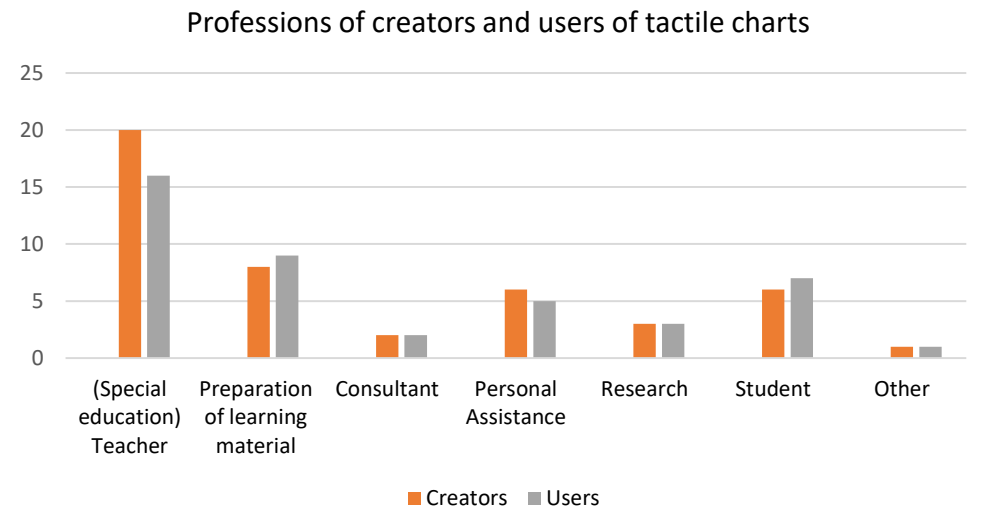

Fig. 1. Number of participants who have experiences in creating (creators) and exploring (users) tactile charts categorized in their profession (in total 55 answers)

\subsection{Production of Tactile Charts}

This section presents results from questions concerning tactile chart production. We have investigated two main research questions: Firstly, who are the creators of tactile charts? Secondly, how is the typical production process organized?

In total, 53 participants $(75 \%)$ do have experiences with the creation of charts, including 28 sighted and 25 blind/visually impaired participants. Jointly $48 \%$ create tactile charts at least once a month, $19 \%$ of them just multiple times a week. We not only asked for experiences in creation of tactile charts but also for visual charts in order to find out whether blind/visually impaired people create visual charts on their own. Figure 2 shows the distribution of experiences in terms of visual and tactile charts. The majority of sighted participants create visual as well as tactile charts (75\%). $40 \%$ blind/visually impaired creators produce tactile and none visual charts. In total 18 blind/visually impaired participants have experiences in creating tactile charts, whereas 10 of them just used analog methods (for example by carving paper). So, less blind/visually impaired participants create tactile charts in digital form than sighted authors. In addition, blind and visually impaired participants have significantly less experiences 


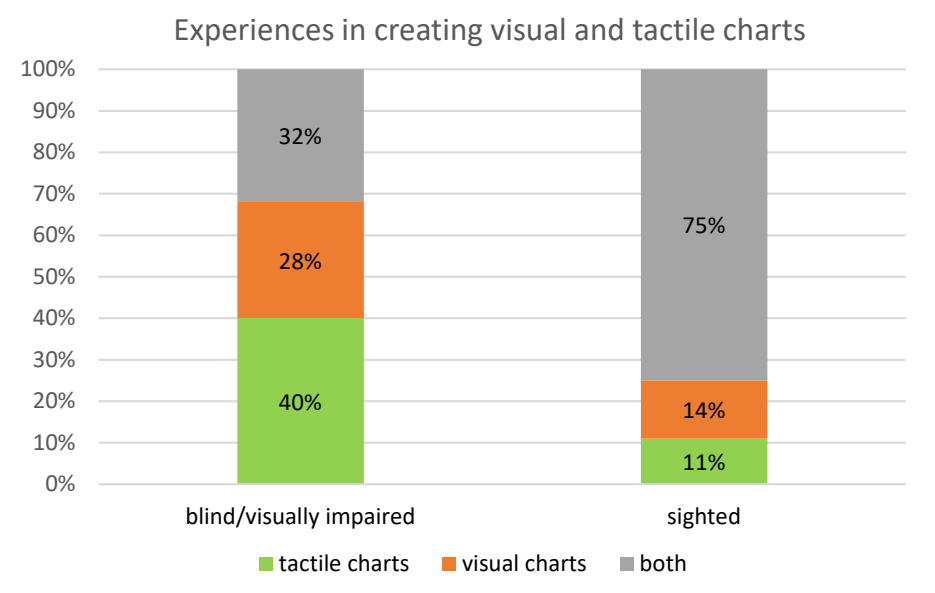

Fig. 2. Distribution of participants (in total 53) who have experiences in creating charts in visual, tactile or both forms. Participants divided into sighted and blind/visually impaired authors.

in creating visual charts $(60 \%)$ than the sighted authors (89\%). Participants who have no experiences (31 with visual charts, 27 with tactile charts) stated two major issues: Firstly, blind/visually impaired participants have problems in handling the required software (13 with visual charts, 13 with tactile charts). Secondly, 14 blind participants do not produce visual charts because they cannot access them independently, whereas 16 participants have no opportunity to print tactile charts. Only six participants have no need to produce charts on their own.

Beyond that, we were interested in the variety of chart types that are created by the participants. Simple bar charts, line and pie charts are well-known and created by more than $90 \%$ of the participants. Around $20 \%$ are familiar with stacked bars, area charts and scatter plots. The remaining group consists of bubble and donut charts as well as star plots which are produced by $10 \%$. More complex chart types are created and known significantly less often than simple chart types.

Afterward, we summarize properties of the production process by presenting most common answers regarding data input, software for creation, steps of adaption, challenging tasks, typical chart elements as well as the target group.

In total, $89 \%$ take a visual chart and $59 \%$ a data table as input. $43 \%$ use Microsoft Office for creating tactile charts. $44 \%$ create a visual chart as basis and adapt it for the tactile output. Typical steps included in the adaption process are translating text labels into Braille $(89 \%)$, creating or adapting a legend (85\%), adapting distances (78\%) and symbol sizes (74\%) as well as the axis scale or change textures and line styles $(70 \%)$. Design criteria for charts based in majority of experiences from the target group (62\%). For $63 \%$ of the participants it is challenging to create tactile charts in an appropriate time. The specification 
of own design criteria (44\%) and the implementation of them (26\%) are also challenging. We asked the participants to name those chart elements a typical chart should contain. The most frequent answers where axis labels $(88 \%)$, a title $(88 \%)$, the axes itself $(83 \%)$, a legend $(76 \%)$ as well as labels in Braille $(71 \%) .67 \%$ of the creators make use of tick marks on the axes while $62 \%$ add precise values. The charts are mainly created for a known target group (for example a school class, $62 \%$ ). $43 \%$ produce charts for individual people. Only seven participants (of 42) create tactile charts for themselves.

With this in mind, the following section derives specific user preferences and requirements for tactile charts.

\subsection{Users of Tactile Charts}

The second part of our survey refers to the context of usage and exploring strategies as well as preferences of the users.

Generally, there are more blind/visually impaired participants (35 equals $85 \%$ ) who have experiences in exploring tactile charts than sighted participants ( 25 equals $67 \%$ ). About half of blind/visually impaired participants explored tactile charts at least once a month within the last year. Eight participants used charts several times a week. The majority get in contact with tactile charts in school as well as in the context of their profession (see Figure 3).

More than half of participants use tactile charts to get access to a visual chart (see Figure 4). $36 \%$ especially blind and visually impaired participants use tactile charts to learn visual concepts. Just as many explore precise values in the chart.
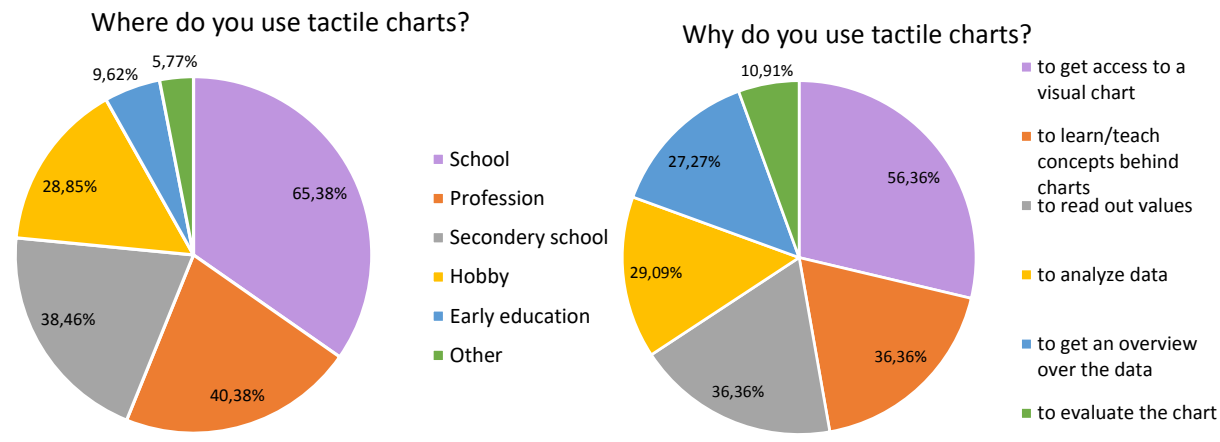

Fig. 3. Usage context of tactile charts.

Fig. 4. Different tasks that participants want to reach with tactile charts.

For exploring tactile charts title $(82 \%)$, legend $(76 \%)$, axis titles $(75 \%)$ and axes $(64 \%)$ are most important. Braille labels $(51 \%)$, tick marks and precise values $(40 \%)$ are essential, too, for analyzing data. Tactile chart design depends on the way how the user interacts with the chart because it should guide him. 
So, we have analyzed 43 free text answers from users of tactile charts. The exploration process can be summarized by following four steps (based on common answers):

1. Getting an overview (14 participants):

- Find out chart type by title (six participants) or initial scan (moving with both hand over the full graphic)

- It can be useful to search for axes and their labels to get an idea of chart content

2. Orientation/Configuration:

- Seeking for general reference points: axes characteristics (eight participants), Braille font, specific symbols, frame of the sheet or legend

\section{Exploration/Getting content:}

- If not already done: exploring of legend, textures and symbols

- Systematic exploration strategies (horizontal or vertical) are used by four participants

- Exploring chart elements like bars, lines or pie segments in detail and read all Braille labels

4. Getting details:

- Searching for guiding elements such as help or grid lines

- Explore precise values (ten participants), estimate element sizes and differences by use of the index finger as reference and exploring with the other hand around it

Some design issues leads to challenges in exploration. Getting too much information $(62 \%)$ is a major problem in tactile chart design. Users should be supported by getting an overview (challenging for $38 \%$ ). Furthermore, it is challenging to keep orientation $(36 \%)$ and to distinguish chart elements from aid lines or grid (35\%). Afterward, we asked how an accessible tactile chart has to be designed (free text answers). In the following we summarize some general results formulated as guidelines in descending order to the number of given answers:

- Reduce data content. Show only as much as data as necessary.

- Give a good overview of the data. Guide the user through your chart.

- Use clear Braille labels (with legend if necessary) with clear assignment to chart elements.

- Omit grid or aid lines if they are dispensable. Otherwise, ensure that they are considerably distinguishable from other elements.

- Different textures, symbols and lines styles should be easily distinguishable and referenced in a legend.

- Support the user by reading out precise values.

- Support access for non-Braille users by overlaying the printed version.

- To support understanding the chart, provide a well written image description.

- Take care that the chart being large enough regarding the size in printed form.

Afterward, we discuss our results and derive properties of target group as well as requirements for a tool that supports authors by creating tactile charts. 


\section{Requirements for a Tool that Automates the Creation of Tactile Charts}

In general, the software addresses two main issues: Firstly, it should support authors of tactile charts by automating the creation process. Secondly, the tool should improve the readability and contribution of tactile charts by supporting well-defined design guidelines. To reach these goals user requirements from the target groups are essential.

\subsection{Context of Use and Use Cases}

Mostly, tactile charts were used and created in the field of education by nonexperts such as teachers. A common use case is to transcribe visual charts from teaching materials to enable blind students to access these charts and learn visual concepts. We assume that time and hardware resources are limited, thus, teachers have to prepare their teaching material next to their regular teaching obligations. Many institutions have only one embosser to print tactile graphics. Hence, different embossers should be supported.

For blind and visually impaired people it is challenging to produce visual charts because they can not control it on their own. In particular, a similar tactile chart is needed to control the visual one. In addition, there are also blind authors who create tactile charts on the basis of raw data to analyze them. Therefore, an effective design is needed.

\subsection{User Requirements and Preferences}

Accessible software is a major requirement for blind/visually impaired people. Therefore, it should be possible to handle the software just using a keyboard. The creation should be automated without the need of manual correction based on rich customization features if required.

The resulting charts should be accessible by all target group members. They should support Braille readers (majority of blind users) as well as a high-contrast version for visually impaired. In addition, a visual version of the chart should be included because most of the sighted authors and teachers are not familiar with Braille reading.

\subsection{User Tasks}

A tool for automating the creation of tactile charts should at least support established chart types such as bar, line and pie charts as well as scatter plots. To support blind authors who are not familiar with the offered chart types, it may be useful to include some exemplary tactile charts with a description. The main requirement is the automatic translation and positioning of Braille labels. In particular, users want to be supported by positioning elements, reducing the complexity of the chart without losing content, choosing high distinguishable 
textures, line styles and symbols as well as adding a legend for the whole graphic. Resulting charts should be standardized as well as highly adaptable. The tool should offer different design templates based on underlying guidelines that can be changed by the user to support different use cases. To support user experiences the tool should accept established data formats such as CSV files or direct data input. In addition, transcribing charts that were created with other chart tools (e.g. Excel) should integrate the tactile chart production in the current workflow. Therefore, an interchange format for tactile charts is needed.

\section{Conclusion}

In general, the survey indicates the need for supporting authors and users of tactile charts. We identified the target group, common chart types, as well as a common workflow for creating tactile charts including challenges. In addition, we identified four main steps for exploring tactile charts and formulated requirements for tactile chart design. Based on the survey we have characterized requirements for the automation application. It should not only decrease the production time but also improve the quality of the charts. They can be produced and evaluated on the basis of well-defined guidelines. Thus, tactile charts can be created with a consistent design which facilitates the exploration. Furthermore, we not only want to support default chart designs but also features to enhance the effectiveness of charts such as different grid types or help lines. We are currently investigating further studies concerning effective tactile chart design for the development of suitable guidelines that can be integrated into the tool. Furthermore, we will improve chart accessibility by adding audio output. Therefore we will explore different interaction strategies and technologies.

In the following developing step we will identify a whole concept and architecture for the automating application that reaches the identified requirements. In addition, we investigate user studies for effective design of tactile charts and interacting concepts. As a final conclusion, we want to improve the availability of tactile charts by enhancing the creating process as well as the quality of tactile charts. So, we want to enable blind and visually impaired people to analyze data as effortless as possible to support the inclusion of disabled people.

\section{Acknowledgment}

The paper is part of the Mosaik project, which is sponsored by the German Federal Ministry of Labour and Social Affairs (BMAS) under the grant number 01KM151112. Only the authors of this paper are responsible for its content.

\section{References}

1. Araki, K., Watanabe, T., Minatani, K.: Development of tactile graph generation software using the $\mathrm{R}$ statistics software environment. Proceedings of the 16th International ACM SIGACCESS Conference on Computers \& Accessibility pp. 251-252 (2014) 
2. Ault, H.K., Deloge, J.W., Lapp, R.W., Morgan, M.J., Barnett, J.R.: Evaluation of Long Descriptions of Statistical Graphics for Blind and Low Vision Users. Mechanical Engineering pp. 25-32 (2002)

3. Braille Authority of North America, Canadian Braille Authority: Guidelines and Standards for Tactile Graphics (2011)

4. Demir, S., Carberry, S., Mccoy, K.F.: Generating Textual Summaries of Bar Charts. Fifth International Natural Language Generation Conference pp. 7-15 (2007)

5. Demir, S., Oliver, D., Schwartz, E., Elzer, S., Carberry, S., McCoy, K.F.: Interactive SIGHT into information graphics. Proceedings of the 2010 International Cross Disciplinary Conference on Web Accessibility (W4A) - W4A '10 p. 1 (2010)

6. Disabilities, R.T.o.I.A.f.P.w.P.I.: Round Table on Information Access Guidelines on Conveying Visual Information (2005)

7. Goncu, C., Marriott, K.: Tactile chart generation tool. Proceedings of the 10th international ACM SIGACCESS conference on Computers and accessibility - Assets '08 p. 255 (2008)

8. Goncu, C., Marriott, K., Hurst, J.: Usability of accessible bar charts. Lecture Notes in Computer Science (including subseries Lecture Notes in Artificial Intelligence and Lecture Notes in Bioinformatics) 6170 LNAI, 167-181 (2010)

9. Jayant, C., Renzelmann, M., Wen, D., Krisnandi, S., Ladner, R., Comden, D.: Automated tactile graphics translation. Proceedings of the 9th international ACM SIGACCESS conference on Computers and accessibility - Assets '07 p. 75 (2007)

10. Keim, D.: Information visualization and visual data mining. IEEE Transactions on Visualization and Computer Graphics 8(1), 1-8 (2002)

11. Moraes, P., Sina, G., McCoy, K., Carberry, S.: Evaluating the accessibility of line graphs through textual summaries for visually impaired users. Proceedings of the 16th international ACM SIGACCESS conference on Computers \& accessibility ASSETS '14 pp. 83-90 (2014)

12. Moraes, P.S., Carberry, S., McCoy, K.: Providing access to the high-level content of line graphs from online popular media. Proceedings of the 10th International Cross-Disciplinary Conference on Web Accessibility - W4A '13 pp. 1-10 (2013)

13. Panëels, S., Roberts, J.C.: Review of designs for haptic data visualization. IEEE Transactions on Haptics 3(2), 119-137 (2010)

14. Prescher, D., Bornschein, J., Weber, G.: Production of accessible tactile graphics. Lecture Notes in Computer Science (including subseries Lecture Notes in Artificial Intelligence and Lecture Notes in Bioinformatics) 8548 LNCS(PART 2), 26-33 (2014)

15. Watanabe, T., Yamaguchi, T., Nakagawa, M.: Development of software for automatic creation of embossed graphs: Comparison of non-visual data presentation methods and development up-to-date. Lecture Notes in Computer Science (including subseries Lecture Notes in Artificial Intelligence and Lecture Notes in Bioinformatics) 7382 LNCS(PART 1), 174-181 (2012) 